\title{
BMJ Open Protocol for a systematic review of telephone delivered psychosocial interventions on relapse prevention, adherence to psychiatric medication and health risk behaviours in adults with a psychotic disorder
}

\author{
Alison K Beck, ${ }^{1}$ Amanda Baker, ${ }^{1}$ Alyna Turner, ${ }^{1}$ Gillian Haddock, ${ }^{2}$ Peter J Kelly, ${ }^{3}$ \\ Katherine Berry, ${ }^{2}$ Sandra Bucci ${ }^{2}$
}

To cite: Beck AK, Baker A, Turner A, et al. Protocol for a systematic review of telephone delivered psychosocial interventions on relapse prevention, adherence to psychiatric medication and health risk behaviours in adults with a psychotic disorder. BMJ Open 2015;5: e009985. doi:10.1136/ bmjopen-2015-009985

- Prepublication history and additional material is available. To view please visit the journal (http://dx.doi.org/ 10.1136/bmjopen-2015009985).

Received 14 September 2015 Revised 26 October 2015 Accepted 9 November 2015

\section{CrossMark}

For numbered affiliations see end of article.

\section{Correspondence to} Dr Alison Beck; Alison.Beck@newcastle.edu. $\mathrm{au}$

\section{ABSTRACT}

Introduction: The mental and physical health of individuals with a psychotic illness are typically poor. When adhered to, medication can reduce relapse. However, despite adherence, relapse remains common and functional outcomes often remain compromised. Compliance is also typically low. Cardiovascular-related morbidity and mortality is also elevated, along with several important modifiable health risk behaviours. Access to psychosocial interventions is therefore important, but currently limited. Telephone delivered interventions represent a promising solution, although further clarity is needed. Accordingly, we aim to provide an overview and critical analysis of the current state of evidence for telephone delivered psychosocial interventions targeting key health priorities in adults with a psychotic disorder, including (1) relapse, (2) adherence to psychiatric medication and/or (3) modifiable cardiovascular health risk behaviours.

Methods and analysis: Our methods are informed by published guidelines. The review is registered and any protocol amendments will be tracked. Ten electronic peer-reviewed and four grey literature databases have been identified. Preliminary searches have been conducted for literature on psychosocial telephone interventions targeting relapse, medication adherence and/or health risk behaviours in adults with a psychotic disorder. Articles classified as 'evaluation' will be assessed against standardised criteria and checked by an independent assessor. The searches will be re-run just before final analyses and further studies retrieved for inclusion. A narrative synthesis will be reported, structured around intervention type and content, population characteristics and outcomes. Where possible, 'summary of findings' tables will be generated for each comparison. For the primary outcome of each trial, when data are available, we will calculate a risk ratio and its $95 \% \mathrm{Cl}$ (dichotomous outcomes) and/or effect size according to Cohen's formula (continuous outcomes). Ethics and dissemination: No ethical issues are foreseen. Findings will be disseminated widely to clinicians and researchers via journal publication and conference presentation(s).

Trial registration number: PROSPERO CRD42015025402.

\section{INTRODUCTION}

Psychotic illnesses (eg, schizophrenia spectrum and bipolar disorder) are chronic, relapsing conditions characterised by distortions in thinking, perception and emotional response. ${ }^{1}$ These symptoms can have a profound impact on quality of life and functioning. ${ }^{2}$ Psychotic illnesses are also associated with a mortality rate double that of the general population ${ }^{3}{ }^{4}$ and a shortening of life expectancy by up to 19 years. ${ }^{5}$ Cardiovascular disease (CVD) is the single largest cause of death among this group, accounting for more premature deaths than suicide ${ }^{6-8}$ Rates of major health risk behaviours associated with CVD (smoking, physical inactivity, alcohol use and low fruit and vegetable intake) are all higher in people living with psychotic illnesses. ${ }^{9-12}$ Furthermore, second generation antipsychotics (SGA), which are commonly used in the treatment of psychotic illnesses, are also associated with a range of serious metabolic side effects, including changes in body weight, glucose utilisation and lipid status. ${ }^{13}$

The well-being of individuals with psychotic illnesses is further compromised by poor access to treatment. Although SGAs can reduce relapse, ${ }^{14}$ rates of non-compliance are as high as $50 \% .{ }^{15}$ A large scale study has also found that almost three-quarters of 
participants diagnosed with schizophrenia chose to discontinue their medication within 18 months. ${ }^{16}$ Furthermore, for those individuals who are compliant and do benefit from medication, they often continue to experience difficulties within important psychosocial domains (eg, employment, social function) and continue to relapse. ${ }^{2}{ }^{14}$ This points to the importance of psychosocial interventions as an adjunct to traditional medication management.

Cognitive-behavioural therapy (CBT) is one of the most researched psychosocial interventions in psychosis. CBT is associated with small to moderate positive effects for a range of psychotic symptomatology and accompanying difficulties ${ }^{17}$ and also demonstrates promise as an option for improving adherence to antipsychotic medication. ${ }^{19}$ Furthermore, increasing evidence supports the role of CBT alone, or in combination with, other psychosocial approaches (eg, motivational interviewing) for modifying health risk behaviours among individuals with psychosis. ${ }^{20-22}$ However, despite psychosocial interventions like CBT being recommended by Australian, ${ }^{23} \mathrm{UK}^{24}{ }^{25}$ and other international clinical guidelines ${ }^{26} 27$ for the treatment of schizophrenia and other psychotic disorders, of those likely to benefit, only $10 \%$ or less have access. ${ }^{28-30}$ Barriers to access include availability of trained clinicians, accessibility of support services, embarrassment and perceived stigma associated with seeking help. ${ }^{28-30}$ Given the limitations of medication management, improving access to psychosocial interventions represents an important priority for enhancing the well-being of individuals living with a psychotic illness.

\section{Why it is important to do this review}

Technology-based interventions represent a promising avenue for improving access to healthcare. Indeed, a recent systematic reviews points to the acceptability and feasibility of telephone delivered interventions (alone, or in combination with other remote access technology) within schizophrenia. ${ }^{31}$ However, this review was restricted to schizophrenia and did not focus on psychosocial interventions or summarising the evidence for key health priorities. Given that the problems seen in schizophrenia surrounding relapse, SGA compliance, CVD and treatment access are also shared by other psychotic disorders, in this systematic review we aim to provide an overview and critical analysis of the current state of evidence for psychosocial telephone delivered interventions targeting key health priorities in adults with a psychotic disorder, including (1) relapse, (2) adherence to psychiatric medication and/or (3) modifiable cardiovascular health risk behaviours.

\section{Objectives}

The following three questions will be addressed. For adults with a psychotic disorder:

1. Do telephone delivered psychosocial interventions targeting (1) relapse, (2) adherence to psychiatric medication and/or (3) modifiable cardiovascular health risk behaviours result in changes to:

A. Indicators of relapse, including psychiatric symptomatology (positive and negative symptoms, depression, anxiety), the number and duration of hospitalisations, functioning and quality of life

B. Medication adherence, including dose count (doses taken); dose days (davs where correct number of doses taken); dose time (doses taken on schedule)

C. Health behaviours (eg, smoking, substance use, physical activity, fruit and vegetable consumption)

D. Severity of cardiovascular disease (CVD) risk, including CVD risk index; quantity, severity of CVD risk factors (eg, weight, body mass index (BMI), waist circumference, blood pressure, plasma lipids, insulin and glucose)

2. Is the effect of telephone delivered psychosocial interventions targeting (1) relapse, (2) adherence to psychiatric medication and/or (3) modifiable cardiovascular health risk behaviours on the above listed treatment outcomes influenced by:

A. other intervention components (eg, individual and/or group face-to-face components; supplementary materials; other technology)

B. implementation factors (staff training; intervention fidelity, treatment engagement/adherence)

C. process measures/mediators/mechanisms (eg, cognitive (empowerment/self-efficacy/motivation); behavioural (eg, active coping, including managing urges); relational (eg, therapeutic alliance))

3. What is the evidence for the feasibility of telephone delivered psychosocial interventions for relapse prevention, adherence to psychiatric medication and/or health risk behaviours, including commentary on economic outcomes and service user and/or provider satisfaction.

\section{METHODS AND ANALYSIS}

This systematic review will be informed by published guidelines ${ }^{32}$ and reported according to the Preferred Reporting Items for Systematic Review and Meta-analysis (PRISMA). ${ }^{33}$

\section{Eligibility criteria}

Eligibility of papers for inclusion in the review will be informed by inclusion and exclusion criteria applied to each of the following domains: types of studies, types of participants, types of interventions and comparison conditions, and the outcome measures assessed. Inclusion and any exclusion criteria within each of these domains is described in turn below:

\section{Types of studies}

In accordance with the objective of providing an overview of the current evidence for telephone delivered 
interventions in adults with a psychotic disorder, liberal design criteria will be adopted. The following designs will be included-randomised controlled trials (cluster and parallel design); cross-over trial; case series or casecontrols; one-arm trial; non-randomised trials; crosssectional or cohort studies and case reports. As broad inclusion criteria may increase risk of bias, this will be assessed using the Collaboration's Risk of Bias tool, as described in the Cochrane Handbook for Systematic Review of Interventions $\left({ }^{32}\right.$ detailed under risk of bias assessment below). Qualitative only designs will not be included.

\section{Types of participants}

Studies that include adults $(\geq 18)$ with a psychotic disorder, as defined by any criteria will be included. Diagnosis of study participants may be self-reported or confirmed via clinical interview. Study participants may be residing in the community, rehabilitation, treatment and/or correctional facility. We will include studies with populations involving adults with non-psychotic disorders only if more than $50 \%$ had a psychotic disorder, or if data limited to those with psychotic disorders are available.

In order to better inform research and clinical care, we intend to describe the clinical state (acute vs postacute vs partial remission vs remission), stage (eg, first episode vs early illness vs persistent) and whether the studies target particular clinical presentations (eg, negative symptoms, positive symptoms, treatment-resistant illnesses).

\section{Types of interventions}

The intervention of interest is telephone support targeting (1) relapse prevention, (2) adherence to psychiatric medication and/or (3) modifiable health risk behaviours.

'Relapse prevention' will be defined as telephone support designed to recognise and act on early warning signs of episode recurrence and/or enhance coping strategies (including medication compliance), the number and duration of hospitalisations and/or the impact of the illness on functioning and/or quality of life.

'Adherence to psychiatric medication' will be defined as telephone support intended to affect adherence with prescribed, self-administered medication for mental disorders. Ethical standards for adherence research dictate that attempts to increase adherence must be judged by their clinical benefits, not simply their effects on adherence rates. ${ }^{34}$ Accordingly, adherence studies will only be included if both adherence and treatment effects are measured.

'Modifiable health risk behaviours' will be defined as telephone support that targets health behaviours (nutrition, physical activity, smoking and substance use) associated with modifiable cardiovascular risk factors (weight, cholesterol, blood glucose and blood pressure).
To be included, the telephone support must:

1. Be administered over the telephone using person delivered (professional or layperson) spoken word (ie, text, web-based and/or automated systems collecting or transmitting data will not be included)

2. Utilise one or more psychological strategies to modify relapse risk, adherence to psychiatric medication and/or health risk behaviours. Psychological strategies will be defined as supportive counselling, psychoeducation (including brief advice), cognitive behavioural (including problem solving, dialectical behavioural therapy, acceptance and commitment therapy), mindfulness and/or motivational interviewing

3. Comprise at least one telephone session, of at least $10 \mathrm{~min}$, delivered by a healthcare professional and/ or non-professional/layperson/peer/consumer who has been trained in delivering the intervention

The telephone support may be a standalone intervention and/or delivered in combination with other treatment components, including pharmacological. However, studies with multiple components will only be included if the telephone is the predominant method of intervention delivery. This is defined as studies in which at least $50 \%$ of the total number of participant contacts are conducted by telephone. Interventions delivered in any setting (eg, community, hospital, rehabilitation or residential treatment centre, etc) will be included.

\section{Types of comparison conditions}

The telephone support may be compared to inactive (eg, standard care, waiting list control) and/or active controls (eg, pharmacological and/or psychological) whereby telephone is not the predominant method of intervention delivery (eg, individual, group or internet).

\section{Types of outcome measures}

1. Indicators of relapse, including psychiatric symptomatology (positive and negative symptoms, depression, anxiety), the number and duration of hospitalisations, functioning and quality of life

2. Medication adherence, including dose count (doses taken); dose days (days where correct number of doses taken); dose time (doses taken on schedule)

3. Health behaviours (eg, smoking, substance use, physical activity, fruit and vegetable consumption)

4. Severity of cardiovascular risk, including CVD risk index; quantity, severity of CVD risk factors (eg, weight, BMI, waist circumference, blood pressure, plasma lipids, insulin, glucose)

5. Treatment engagement (eg, quantity/frequency/duration of telephone support attendance)

6. Process measures/mediators/mechanisms (eg, cognitive (empowerment/self-efficacy/motivation); behavioural (eg, active coping, including managing urges); process (eg, therapeutic alliance))

7. Feasibility, including economic outcomes (eg, cost, resource use, cost-effectiveness) and/or satisfaction/ 
preference. Qualitative outcomes regarding participant and/or relative satisfaction will be reported as described.

Outcomes may be clinician and/or patient rated; assessed by objective and/or subjective indices (eg, blood, urine, actigraph, questionnaire, monitoring form/diary) with or without collateral information (eg, using a family member to validate use) and of any time frame (eg, baseline, short and/or medium and/or longterm follow-up).

\section{Information sources}

Search strategy

Consistent with methods detailed in Cochrane Guidelines for systematic reviews, ${ }^{32}$ the search strategy will be conducted as follows. First, in May 2015 we identified 10 relevant scientific electronic databases (MEDLINE, PubMed, EMBASE, CINAHL, Science Direct, Wiley, PsychInfo, Central, Amed, Scopus) and four electronic non-scientific databases (Translating Research into Practice; Virginia Commonwealth University; Project Cork; Prevention, Information and Evidence Library) to search. Search terms related to telephone will be combined with psychosis-related search terms and then outcome-related search terms (see online supplementary appendix 1 for the full MEDLINE search strategy).

Abstract, title, key words and subject headings specific to each of the identified database will be searched. All subject headings will be exploded so that narrower terms are included. No limits will be placed on publication year. Publications must be available in English. Reference lists of identified publications will be manually searched to identify any additional publications. All publications will be organised in reference manager Endnote. The searches will be re-run just before final analyses and further studies retrieved for inclusion.

\section{Classification of studies}

The titles and abstracts of identified references will be classified in a three-step process.

\section{Step 1: identification of studies for exclusion}

AKB will review the titles and/or abstracts and exclude articles if they: (1) are duplicates, (2) do not focus on adults with a psychotic disorder, (3) do not focus on telephone delivered support, or (4) if the outcomes, process and/or predictor variables do not include or specifically relate to relapse, medication adherence and/or health behaviours, (5) are not journal articles, reports, book chapters or newsletter articles. If eligibility is unclear from the title and/or abstract, the full text article will be accessed and assessed.

\section{Step 2: classification of studies}

The abstracts and/or full text of the remaining studies will be examined by AKB to identify studies that are: (1) Evaluation, defined as an evaluation of a telephone delivered intervention as per the PICO criteria outlined above; (2) Reviews, including summaries, descriptive, critical and/or systematic reviews; Discussion, defined as general discussion of telephone delivered interventions, including development, principles, methods and implementation. References that are not evaluation, review or discussion papers (eg, treatment manuals) will classified as 'Other'.

\section{Step 3: cross checking}

Publications from step two will be reclassified by $A B$, for cross-checking. In case of disagreement, the final classification will be made by consensus, with the involvement of GH, PK, KB and/or SB. The articles excluded in step one will not be cross-checked because they will not be relevant to the review. The evaluation studies identified in step two will retained for further examination.

\section{Data extraction from evaluation studies}

Data extraction will be performed by $\mathrm{AB}$ and checked by AT. Extraction forms will be piloted on several papers and modified as needed before use. When multiple reports of the same study are identified (eg, related journal articles, conference proceedings which are then published), data from each report will be extracted separately and then combined across multiple data collection forms. Methodological critique and assessment of risk of bias will be performed independently by $\mathrm{AB}$ and AT. In the event of disagreement, final ratings will be made via consensus, following discussion with GH, PJK, $\mathrm{KB}$ and/or SB. In the event that inadequate trial details are reported, study authors will be contacted no more than twice to obtain further information.

To enable methodological critique of both observational research and RCTs, criteria for data extraction will be adapted from the Downs and Black Scale ${ }^{35}$ and the Cochrane Handbook for Systematic Reviews ${ }^{32}$ and include

1. Participant information, including n-values at each stage of the study (and reasons for nonparticipation), treatment setting, eligibility criteria, descriptive data including age, gender, ethnicity, socioeconomic status, diagnostic criteria and treatment history

2. Methods, including study design, country, setting(s), methodological limitations reported, methodological limitations observed (eg, recruitment allocation and data collection methods; blinding; comparability of groups at baseline; appropriateness of analysis methods)

3. Interventions, including number of groups, duration of treatment (number, frequency and duration of phone and non-phone components), delivery $\operatorname{method}(\mathrm{s})$, description of control intervention(s)

4. Primary and secondary outcomes, including data collection sources/methods, percentage of treatment sessions attended, other process measures/ 
mediators/mechanisms, economic outcomes, satisfaction-related qualitative outcomes, follow-up period

5. Results, including indicators of relapse, medication adherence, health behaviours, severity of cardiovascular risk, treatment engagement, process measures/ mediators/mechanisms, economic outcomes and patient satisfaction collected at all available follow-up time points.

\section{Methodological critique of evaluation research}

To provide a thorough overview of the literature we will implement procedures to evaluate the quality of both observational studies and RCTs. A narrative synthesis of the findings from the included studies will be reported, structured around intervention type and content, population characteristics, and outcomes. This qualitative review will be supplemented with the following quantitative measures.

For observational studies, methodological quality will be assessed against the Downs and Black Scale. ${ }^{35}$ Criteria will be assigned a yes (1 point); no ( 0 points); or unclear $(0$ points $)$ rating. All criteria will have the same weight, and a quality score ranging from 0 to 27 points will be calculated for each study.

For RCTs, methodological quality will be assessed against the 11-item Physiotherapy Evidence Database (PEDro) scale. ${ }^{36}$ Consistent with published reviews of psychological interventions ${ }^{21} 37$ two items regarding blinding of participants and therapists will not be scored in the present review, as these criteria are not appropriate for the studies under review. The remaining nine criteria will be assigned a assigned a yes ( 1 point) or no $(0$ points) rating, and a quality score ranging from 0 to 8 points will be calculated for each study (as item one is not included in the quality score ${ }^{36}$;).

Risk of bias will also be assessed using the Collaboration's Risk of Bias tool, as described in the Cochrane Handbook for Systematic Review of Interventions. ${ }^{32}$ We will judge each item as being high, low or unclear risk, as per the criteria provided by Higgins and Green ${ }^{32}$ and provide a quote from the study report and a justification for our judgement for each item in the risk of bias table. Given that growing empirical evidence suggests that sequence generation and allocation concealment are particularly important potential sources of bias, studies will be deemed to be at the highest risk of bias if either item is scored as 'high' or 'unclear'.

\section{Measures of treatment effect}

Where possible, 'summary of findings' (SOF) tables will be generated for each comparison (phone vs multicomponent phone; phone vs other active control; phone vs other inactive control). SOF tables will provide key information regarding evidence quality, the magnitude of effect of the interventions examined, and a summary of available data on the outcome variables defined under 'Outcome Measures' above.
Scale-derived data

We intend to include continuous data from rating scales only if:

A. The psychometric properties of the instrument have been described in a peer-review journal

B. The instrument was not written or modified by one of the authors for that particular trial

C. The instrument was self-report or completed by an independent assessor (in the event that this is not clearly reported, a note will be made in 'Description of Studies')

\section{Data presented in graphs and figures}

Where possible, we intend to extract data that is only represented in graphs and figures, but only if the same result(s) are independently derived by $\mathrm{AB}$ and $\mathrm{AT}$.

\section{Dichotomous outcome measures}

When data are available, a risk ratio (RR) and its $95 \%$ CI will be provided for the primary outcome of each trial. RR has been selected in preference to ORs as evidence suggests that $R R$ is more intuitive ${ }^{38}$ and clinicians tend to misinterpret ORs as RR. ${ }^{39}$

\section{Continuous outcome measures}

When data are available, effect sizes will be calculated according to Cohen's formula, to allow for comparison across studies. Effect sizes will be interpreted according to published guidelines, where $0.2-0.49$ is defined as a small effect size, $0.5-0.79$ is moderate and greater than 0.8 is large.

A study will be considered to have a positive outcome if at least $50 \%$ of reported outcomes demonstrate a between-group difference in favour of the telephone support group at the end of the intervention. Positive maintenance outcome(s) will be evidenced when this effect is also evident at short and/or medium and/or long-term follow-up (defined as 1-6; 7-12 and $>12$ months after intervention completion, respectively). We anticipate there will be limited scope for meta-analysis due to the range of different outcome measures.

\section{ETHICS AND DISSEMINATION}

As no primary data collection will be undertaken, no formal ethical assessment is required.

We plan to present the findings of this systematic review for peer-review in an appropriate journal. We also intend to present to clinicians and researchers at appropriate conferences.

\section{Author affiliations}

${ }^{1}$ School of Medicine and Public Health, University of Newcastle, Callaghan, New South Wales, Australia

${ }^{2}$ School of Psychological Sciences, The University of Manchester, Manchester, UK

${ }^{3}$ School of Psychology, University of Wollongong, Wollongong, New South Wales, Australia 
Contributors $A B$ is the guarantor of the review protocol and wrote the draft protocol for the systematic review. All authors made substantial contributions to the conception and design of this protocol, revising it critically for important intellectual content and gave final approval of the version to be published. All authors agree to be accountable for all aspects of the work to ensure that questions related to the accuracy or integrity of any part of the work are appropriately investigated and resolved.

Funding Funding support for the conduct of this review has been provided by the NHMRC Centre of Research Excellence for Mental Health and Substance Use. The funder has no involvement in developing this protocol.

Competing interests $A K B, A B, A T$ and PJK have no competing interests to declare. SB, KB and GH are current grant holders for a mobile application delivered CBT intervention for early psychosis (Medical Research Council: R116690).

\section{Patient consent Not obtained.}

Provenance and peer review Not commissioned; externally peer reviewed.

Open Access This is an Open Access article distributed in accordance with the Creative Commons Attribution Non Commercial (CC BY-NC 4.0) license which permits others to distribute, remix, adapt, build upon this work noncommercially, and license their derivative works on different terms, provided the original work is properly cited and the use is non-commercial. See: http:// creativecommons.org/licenses/by-nc/4.0/

\section{REFERENCES}

1. Morgan VA, Waterreus A, Jablensky A, et al. People living with psychotic illness in 2010: the second Australian national survey of psychosis. Aust N Z J Psychiatry 2012;46:735-52.

2. Brissos S, Dias VV, Balanza-Martinez V, et al. Symptomatic remission in schizophrenia patients: relationship with social functioning, quality of life, and neurocognitive performance. Schizophr Res 2011;129:133-6.

3. Brown S, Kim M, Mitchell C, et al. Twenty-five year mortality of a community cohort with schizophrenia. Br J Psychiatry 2010;196:116-21.

4. Saha S, Chant D, McGrath J. A systematic review of mortality in schizophrenia: is the differential mortality gap worsening over time? Arch Gen Psychiatry 2007;64:1123-31.

5. Laursen TM. Life expectancy among persons with schizophrenia or bipolar affective disorder. Schizophr Res 2011;131:101-4.

6. Brown S, Barraclough B, Inskip $\mathrm{H}$. Causes of the excess mortality of schizophrenia. BR J Psychiatry 2000;177:212-17.

7. Bushe CJ, Taylor M, Haukka J. Mortality in schizophrenia: a measurable clinical endpoint. J Psychopharmacol 2010;24(4 Suppl):17-25.

8. De Hert M, Dekker JM, Wood D, et al. Cardiovascular disease and diabetes in people with severe mental illness position statement from the European Psychiatric Association (EPA), supported by the European Association for the Study of Diabetes (EASD) and the European Society of Cardiology (ESC). Eur Psychiatry 2009;24:412-24.

9. Galletly CA, Foley DL, Waterreus A, et al. Cardiometabolic risk factors in people with psychotic disorders: the second Australian national survey of psychosis. Aust N Z J Psychiatry 2012;46:753-61.

10. Morgan VA, Waterreus A, Jablensky A, et al. People living with psychotic illness in 2010: the second Australian national survey of psychosis. Aust N Z J Psychiatry 2012;46:735.

11. Australian Bureau of Statistics. Australian health survey: first results, 2011-2012. Canberra, Australia: Australian Bureau of Statistics, 2012. (Cat. No.4364.0.55.001)

12. Teesson $\mathrm{M}$, Hall $\mathrm{W}$, Slade $\mathrm{T}$, et al. Prevalence and correlates of DSM-IV alcohol abuse and dependence in Australia: findings of the 2007 National Survey of Mental Health and Wellbeing. Addiction 2010;105:2085-94.

13. Rummel-Kluge C, Komossa K, Schwarz S, et al. Head-to-head comparisons of metabolic side effects of second generation antipsychotics in the treatment of schizophrenia: a systematic review and meta-analysis. Schizophr Res 2010;123:225-33.

14. Alvarez-Jimenez M, Priede A, Hetrick SE, et al. Risk factors for relapse following treatment for first episode psychosis: a systematic review and meta-analysis of longitudinal studies. Schizophr Res 2012;139:116-28.

15. Lacro JP, Dunn LB, Dolder CR. Prevalence of and risk factors for medication nonadherence in patients with schizophrenia: a comprehensive review of recent literature. J Clin Psychiatry 2002;63:892-909.
16. Lieberman JA, Stroup TS, McEvoy JP, et al. Effectiveness of antipsychotic drugs in patients with chronic schizophrenia. $N$ Engl J Med 2005;353:1209-23.

17. Wykes T, Steel C, Everitt B, et al. Cognitive behavior therapy for schizophrenia: effect sizes, clinical models and methodological rigor. Schizophr Bull 2008;34:523-37.

18. Jauhar S, McKenna PJ, Radua J, et al. Cognitive-behavioural therapy for the symptoms of schizophrenia: systematic review and meta-analysis with examination of potential bias. Br J Psychiatry 2014;204:20-9.

19. Barkhof E, Meijer CJ, de Sonneville LM, et al. Interventions to improve adherence to antipsychotic medication in patients with schizophreniaa review of the past decade. Eur Psychiatry 2012;27:9-18.

20. Banham L, Gilbody S. Smoking cessation in severe mental illness: what works? Addiction 2010;105:1176-89.

21. Baker AL, Hiles SA, Thornton LK, et al. A systematic review of psychological interventions for excessive alcohol consumption among people with psychotic disorders. Acta Psychiatr Scand 2010;126:243-55.

22. Baker A, Richmond R, Castle D, et al. Coronary heart disease risk reduction intervention among overweight smokers with a psychotic disorder: pilot trial. Aust N Z J Psychiatry 2009;43:129-35.

23. Royal Australian and New Zealand College of Psychiatrists Clinical Practice Guidelines Team for the Treatment of Schizophrenia and Related Disorders. Royal Australian and New Zealand College of Psychiatrists clinical practice guidelines for the treatment of schizophrenia and related disorders. Aust N Z J Psychiatry 2005;39:1-2.

24. National Institute for Health and Care Excellence. Psychosis and schizophrenia in adults: treatment and management NICE guidelines [CG178]. February 2014. https://www.nice.org.uk/ guidance/cg178 (accessed 26 Aug 2015).

25. National Institute for Health and Care Excellence. Bipolar disorder: the assessment and management of bipolar disorder in adults, children and young people in primary and secondary care. NICE guidelines [CG185]. September 2014. https://www.nice.org.uk/ guidance/cg185 (accessed 26 Aug 2015).

26. Dixon L, Perkins D, Calmes C. Guideline watch (September 2009): practice guideline for the treatment of patients with schizophrenia. American Psychiatric Association, 2009. http://psychiatryonline.org/ guidelines (accessed 10 Sep 2015).

27. Hirschfield RMA. Guideline watch: practice guideline for the treatment of patients with bipolar disorder. 2nd edn. American Psychiatric Association, 2005. http://psychiatryonline.org/guidelines (accessed 10 Sep 2015)

28. Schizophrenia Commission. The abandoned illness: a report by the schizophrenia commission. London: Rethink Mental IIIness. http:// www.rethink.org/about-us/the-schizophrenia-commission (accessed 27 Aug 2015)

29. Haddock $G$, Eisner $E$, Boone $C$, et al. An investigation of the implementation of NICE recommended CBT interventions for people for schizophrenia. J Ment Health 2014;23:162-5.

30. Gulliver A, Griffiths KM, Christensen H. Perceived barriers and facilitators to mental health help-seeking in young people: a systematic review. BMC Psychiatry 2010;10:113.

31. Kasckow J, Felmet K, Appelt C, et al. Telepsychiatry in the assessment and treatment of schizophrenia. Clin Schizophr Relat Psychoses 2014;8:21-27A.

32. Higgins JPT, Green S. Cochrane Handbook for Systematic Reviews of Interventions Version 5.1.0 [updated March 2011]. http:// handbook.cochrane.org/ (accessed 5 May 2015).

33. Moher D, Liberati A, Tetzlaff J, et al. Preferred reporting items for systematic reviews and meta-analyses: the PRISMA statement. PLoS Med 2009;6:e1000097.

34. BRIAN R. Management of patient compliance in the treatment of hypertension. Report of the NHLBI Working Group. Hypertension 1982;4:415-23.

35. Downs $\mathrm{SH}$, Black N. The feasibility of creating a checklist for the assessment of the methodological quality both of randomised and non-randomised studies of health care interventions. J Epidemiol Community Health 1998;52:377-84.

36. Centre for Evidence-Based Physiotherapy. PEDro Scale. Centre for Evidence-Based Physiotherapy. Updated 2009. http://www.pedro. org.au

37. Spring B, Howe D, Berendsen $\mathrm{M}$, et al. Behavioral intervention to promote smoking cessation and prevent weight gain: a systematic review and meta-analysis. Addiction 2009;104:1472-86.

38. Boissel JP, Cucherat M, Li W, et al. The problem of therapeutic efficacy indices. 3. Comparison of the indices and their use. Therapie 1999;54:405-11.

39. Deeks J. Issues in the selection for meta-analyses of binary data. Abstracts of 8th International Cochrane Colloquium. 2000 Oct 2528th: Cape Town, South Africa 